\title{
Porosity of Low- $\kappa$ Materials Studied by Slow Positron Beam
}

\author{
R.S. Brusa, C. Macchi, S. Mariazzi and G.P. Karwasz \\ Dipartimento di Fisica, Università di Trento, 38050 Povo (Tn), Italy
}

\begin{abstract}
The information about porosity in low- $\kappa$ materials obtainable by depth profiling with positron annihilation spectroscopy is reviewed. In particular we focus on Doppler broadening spectroscopy and 2-3 $\gamma$ ratio of positronium measurements on $\mathrm{SiOCH}$ and amorphous carbon a-C:F:H thin films produced by plasma enhanced chemical vapour deposition.
\end{abstract}

PACS numbers: 71.60.+z, 78.70.Bj, 68.60.Dv, 68.90.+g

\section{Introduction}

Until the past few years, aluminium was used in semiconductor technology as interconnect metal and silicon dioxide $\left(\mathrm{SiO}_{2}, \kappa=3.9\right)$ as the inter- and intra-level insulator. With the demand of scaling the dimension of advanced integrated circuits well below the $100 \mathrm{~nm}$ DRAM $1 / 2$ pitch technology node [1], the signal delay (resistance-capacitance RC delay) becomes one limitation in increasing the speed of the electronic devices. To reduce the RC delay, materials with lower electrical resistance and lower dielectric constant are needed. Copper is replacing aluminium as interconnect metallization [2] for all the advanced integrated circuit. On the other side, recently, some low- $\kappa$ materials are introduced in electronic devices as insulator in substitutions to $\mathrm{SiO}_{2}$. According to [1] one of the three top interconnect difficult challenges to reach the $45 \mathrm{~nm}$ technology node in the next five years is: "Introduction of new materials to meet conductivity requirements and reduce the dielectric permittivity". The goal is to reduce the dielectric constant below 2. The dielectric constant can be reduced by introducing open space inside the material (pores) and trying to have the skeleton around the pores formed by less polarizable molecules. Total polarization $\bar{P}$, in fact, depends through the following equation on $N$ (number of molecules for $\mathrm{m}^{3}$ ), on electronic $\left(\alpha_{\mathrm{e}}\right)$ polarizability, displacement $\left(\alpha_{\mathrm{d}}\right)$ polarizability and on the term $p_{0} / 3 k T$ due to thermal averaging of the dipole moment of molecules, with permanent dipole moment $p_{0}$, 
accounting for the orientation polarizability:

$$
\bar{P}=N\left(\alpha_{\mathrm{e}}+\alpha_{\mathrm{d}}+\frac{p_{0}}{3 k T}\right) \bar{E}_{\mathrm{loc}},
$$

$\bar{E}_{\text {loc }}$ is the local electric field. The dielectric constant $\kappa$ is related to the total polarizability with the equation:

$$
\frac{\kappa-1}{\kappa+2}=\frac{N}{3 \varepsilon_{0}}\left(\alpha_{\mathrm{e}}+\alpha_{\mathrm{d}}+\frac{p_{0}}{3 k T}\right) .
$$

In the development of new low- $\kappa$ films with thickness of some hundred nanometers, precise characterizations of the structure, pores, skeleton and internal surface of the pore, are needed. Depth profiling with positron annihilation spectroscopy (DP-PAS) is one among the few techniques that can give valuable information on the porosity and, in combination with other techniques for skeleton characterization (vibrational spectroscopy and electrical measurements), can help to have a complete picture of the materials characteristics.

In the following we shall introduce briefly the type of materials used and the production techniques of low- $\kappa$. In this second section we shall also indicate which type of materials have been studied by positron techniques up to now. In the third section, we shall illustrate the information about porosity obtainable by two DP-PAS techniques: the Doppler broadening spectroscopy (DBS) and 2-3 $\gamma$ ratio


will be reported. For an extended and recent review on low- $\kappa$ materials the reader is referred to Ref. [3]. Good description of all the DP-PAS techniques, including positron annihilation lifetime spectroscopy (PALS) and critical comments on the potentiality and limitation of the positron techniques are reported in Refs. [4-7].

\section{Low- $\kappa$}

\subsection{Materials}

At present we classify low- $\kappa$ materials in four main classes:

(i) Silsesquioxane (SSQ) based materials. These organic-inorganic polymers have a ladder-type structure and a cage structure with formula $\left(\mathrm{R}-\mathrm{SiO}_{3 / 2}\right)_{n}$. Eight silicon atoms are placed at the vertex of a cube. For microelectronics applications the hydrogen-silsesquioxane (HSSQ) with $\mathrm{R}=\mathrm{H}$ and methyl-silsesquioxane (MSSQ) with $\mathrm{R}=\mathrm{CH}_{3}$ are the most widely used. With positrons MSSQ materials were studied by DP-PAS with DBS [8, 9] and PALS [10-12]; HSSQ materials by DP-PAS with DBS $[8,13,14]$ and PALS [14].

(ii) Silica based. They have a tetrahedral basic structure of $\mathrm{SiO}_{2}$ in which an $\mathrm{O}$ atom is substituted by $\mathrm{F}$ or $\mathrm{CH}_{3}$ groups. $\mathrm{C}$ bonds are less polarizable than $\mathrm{Si}-\mathrm{O}$ bonds, besides the introduction of $\mathrm{F}$ and $\mathrm{CH}_{3}$ groups increase the free volume of silica. In the case of the introduction of $\mathrm{CH}_{3}$, long chains with different degree of cross linking are formed. The final structure is an open structure with pores. Sputtered $\mathrm{SiO}_{x}$ films [15-17] and $\mathrm{SiOCH}$ [18-21] were studied by positrons. 
(iii) Organic polymers are a wide class of materials consisting of polar and non-polar polymers. We can cite for example benzo-ciclo-butene (BCB) [22]. These materials generally become unstable at temperature above $300-400^{\circ} \mathrm{C}$ and they are also incompatible with the present technological processes. At our knowledge no positron studies have been carried on these materials.

(iv) Amorphous carbon, hydrogenated and/or fluorinated carbon. In these materials it is difficult to obtain very low- $\kappa$ maintaining good mechanical and thermal stability. At our knowledge, as for organic polymers, no positron studies have been done on these materials.

\subsection{Production}

Two main techniques are used to produce low- $\kappa$ materials: spin-on, chemical vapour deposition (CVD) and plasma enhanced chemical vapour deposition (PECVD). HSSQ, MSSQ, and organic polymers are produced by spin-on while silica based materials and amorphous carbon by CVD and PECVD.

In spin-on, a liquid precursor is put at a centre of a substrate on a spinner, usually at room temperature and at atmospheric pressure. By rotating the substrate a uniform film is obtainable. A first treatment at $250^{\circ} \mathrm{C}$ is done to remove the solvent and a second treatment (cure) at $300-600^{\circ} \mathrm{C}$ is done to stabilize the film and to induce the cross linking of the polymer chains.

In CVD and PECVD the precursors are inserted as gas phases in the discharge region between anode (the substrate for low- $\kappa$ deposition) and cathode. With PECVD plasma is formed in the deposition chamber by applying a radiofrequency. The characteristics of the deposited films, porosity and structure, depend on many parameters: precursors, gas pressure, gas temperature, bias, applied voltage. Also after such deposition a thermal treatments of $400-500^{\circ} \mathrm{C}$ is usually necessary to stabilize the materials: formation of cross linking and release of some molecular groups such as $\mathrm{OH}$.

After deposition, other plasma treatments can be necessary to improve the adherence of further layer or to produce a capping layer for sealing the pores. To increase the porosity for approaching $\kappa$ values equal or less than 2 , subtractive molecules are added to the materials during production and removed with thermal treatments. In spin-on macromolecular porogen is added while in CVD a multiphase deposition is used [23].

\subsection{Requirements}

The low- $\kappa$ materials must have a good thermal stability up to $500^{\circ} \mathrm{C}$ and good mechanical properties (adhesion, hardness). This is fundamental to minimize the degradation of the dielectric films during the several processing steps needed to produce a device. Low- $\kappa$ needs to be compatible with $\mathrm{Cu}$ and gases used in plasma treatments. Breakdown voltage is also important. 


\subsection{Porosity}

In order to decrease the dielectric constant it is important to realize materials with a high total porosity. But equal total porosity can be obtained with different size-concentration of the pore types. Besides, the properties of the films are strongly influenced by the type of structure formed by pore and skeleton. We can list the following characteristics that influence the final properties of the films: shape of the pores, size of the pores, connectivity of the pores, distribution and heterogeneity, close or open porosity (in respect to the surface). All these features must be characterized.

\subsection{Techniques for pore characterizations}

There are few techniques for pore characterization, each of them has advantages and drawback, but all of them are model dependent: the information about porosity is obtained by applying some model to the experimental data. We mention: small angle neutron scattering (SANS) and small angle X-ray scattering (SAXS) used in combination with X-ray reflectivity (XRR), ellipsometric porosimetry (EP) and various techniques of DP-PAS: PALS, DBS, $3 \gamma$-PAS. For a description and comparison of these methods we refer to Ref. [3]. The limitation of the different techniques for the study of thin dielectric films is also discussed there. Despite the model dependence, comparative studies carried out on the same type of samples with different techniques (DP-PAS, SAXS, EP) have shown a satisfactory agreement [3]. DP-PAS is the technique more suited for detecting pores of small size.

\section{DBS and $3 \gamma$-PAS}

The equipment required to study low- $\kappa$ films by DP-PAS with DBS and $3 \gamma$-PAS is a slow positron beam tunable in the $0.05-30 \mathrm{keV}$ positron energy range coupled to a high purity germanium detector (HpGe). If coincidence measurements must be performed, two HpGe are necessary. Our set-up consists of an electrostatic positron beam $[24,25]$ that for the present measurements was coupled to an HpGe with an energy resolution of $1.2 \mathrm{keV}$ at $511 \mathrm{keV}$. With $1 \mu \mathrm{m}$ single-crystal tungsten moderator annealed to more than $2000^{\circ} \mathrm{C}$ in situ (starting vacuum $\left.4 \times 10^{-9} \mathrm{mbar}\right)$, about $1000 \mathrm{e}^{+} /(\mathrm{s} \mathrm{mCi})$ are obtained at the target. With HpGe the spectrum of $511 \mathrm{keV}$ annihilation line and of the $356 \mathrm{keV}$ line of $\mathrm{Ba}^{133}$ are recorded simultaneously. With the two lines the $\mathrm{eV} /$ channel is calculated [26]. The annihilation spectrum recorded at each positron implantation energy $E$, is then analyzed according to the following parameters: annihilation with low momentum electrons $(S(E)$ parameter; ratio between the counts in a central area of the $511 \mathrm{keV}$ peak and the total area of the peak); annihilation with high momentum electrons $(W(E)$ parameter; ratio between the wing area of the peak and the 
total area of the peak); the 2-3 $\gamma$ ratio calculated as a ratio between the valley area (about between $410-500 \mathrm{keV}$ ) and the 511 peak area $(R(E)$ parameter). For the choice of the energy windows for $S, W$, and $R$ evaluation, see for example [19, 26]. The combination of $S(E)$ and $W(E)$ in the $S(E)$ vs. $W(E)$ plot [27] point out $S, W$ pairs that are characteristic of the positron annihilation site. In Fig. 1a, b and Fig. 2 we report, as an example, $S, R_{n}$ (normalized $R$ parameter, see below) and $S-W$ curves for SiOCH samples produced by PECVD. All the details about deposition and measurements can be found in Refs. [19-21]. The low- $\kappa$ films were

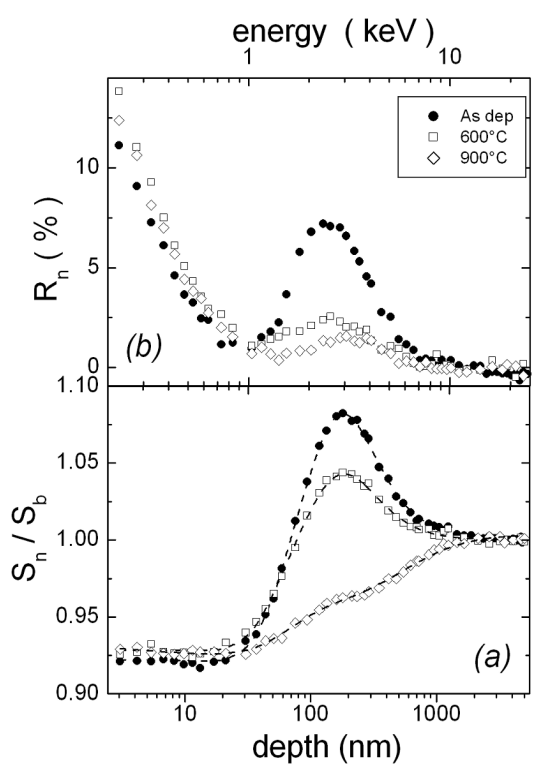

Fig. 1. (a) $S_{n}$ parameter and (b) $R_{n}$ parameter as a function of mean positron implantation depth for SiOCH samples: as-deposited, annealed at 600 and $900^{\circ} \mathrm{C}$ (Ref. $[19,21]$ ).

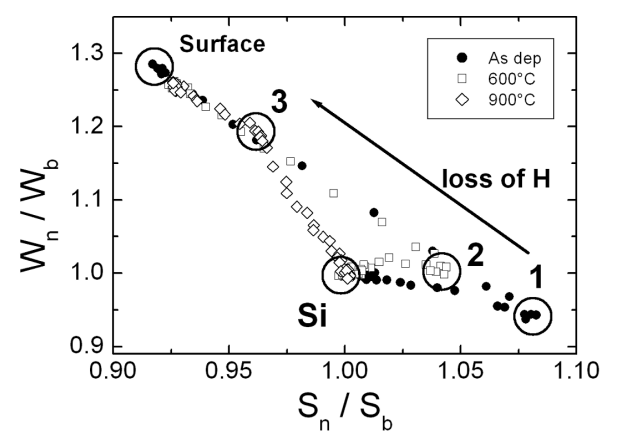

Fig. 2. $S_{n}(E)-W_{n}(E)$ plot for the $\mathrm{SiOCH}$ samples of Fig. 1. Numbers 1, 2, 3 mark the $\mathrm{SiOCH}$ film characteristic point that moves on a strait line as the content of $\mathrm{H}$ decreases (arrow in the figure) with the thermal treatments (Ref. [19, 21]). 
capped by a $\mathrm{N}_{2}+$ He plasma treatment after the annealing at 400 or $480^{\circ} \mathrm{C}$. The data in Fig. 1a, fitted by VEPFIT, show a first layer indicating the capping and the increase in $S$ due to open volumes in the film. Thermal treatments of the film at $600^{\circ} \mathrm{C}$ and $900^{\circ} \mathrm{C}$ produce a decrease in the $S$ value of the film. In Fig. 1b it is evident that the formation of ortho-positronium (o-Ps) in the film, pointing out the presence of pores. It is also clear that capping does not allow the Ps escape from the film: the strong increase of $3 \gamma$ annihilation below $1 \mathrm{keV}$ positron implantation energy, is mainly due to epithermal positrons that are energetically favourite to knock out an electron at the surface and to form free Ps. In Fig. 2, with the thermal treatments, the characteristic $S-W$ point of positrons annihilating in the film moves towards lower $S$ and higher $W$, typical of annihilation with $\mathrm{O}$ and $\mathrm{C}$ atoms. Indeed, by measurements using nuclear techniques, it was found that $\mathrm{H}$ escapes from the samples by increasing the annealing temperature. Most probably the $\mathrm{H}$ is lost from the internal wall of the pores. With the loss of $\mathrm{H}$ there is also formation of $\mathrm{C}$ agglomerates as seen by Raman spectroscopy [21] and at $900^{\circ} \mathrm{C}$ PAS probe the collapsing of the smallest pores.

It is important to underline some details regarding the evaluation of the above parameters $(S, W)$ when the $o$-Ps is formed in a meaningful fraction. The energy of the $3 \gamma$ which originate from the $o$-Ps self annihilation, range from 0 to $511 \mathrm{keV}[28]$. When a significant quantity of $o$-Ps is formed, the left part of the $511 \mathrm{keV}$ annihilation line is distorted. This was not the case of the above measurements where the $o$-Ps fraction annihilating into $3 \gamma$ is at maximum $7 \%$. Otherwise the $S$ and $W$ parameter must be evaluated taking into account only the right half part of the annihilation peak. Another solution is to perform coincidence measurements for $S$ and $W$ evaluation. Annihilation of free positrons, para-positronium $(p-\mathrm{Ps})$ annihilation and pick-off of $o$-Ps contribute to the $S$ parameter.

To have a quantitative measurement of the $3 \gamma o$-Ps decay it is necessary to calibrate the scale of the $R$ parameter. Two procedures can be followed: (a) A sample, in which it is precisely known (by lifetime measurements) the fraction of the $o$-Ps decaying into $3 \gamma$, is used as a standard. In Ref. [15] a sample of silica aerogel was used: (b) The Ps formation is measured in a hot Ge crystal. When the Ge crystal is at about $1000 \mathrm{~K}$, all positrons reaching the surface form positronium [29-31]. On the contrary positrons implanted at high implantation energy do not form Ps in Ge. It is assumed $R_{100}$ ( $100 \%$ positronium formation) as the $R$ value obtained by extrapolating to zero implantation energy $R$ measured in Ge at high temperature. $R_{0}$ is the $R$ value at the highest positron implantation energy. The calibration measurements do require to be done in the same geometrical configuration used for the measurements. Our calibration measurements, by the method (b), are reported in Fig. 3. To check the $100 \%$ positronium formation at high temperature we have calculated the positronium fraction $F$ with the formula $[29,30]: F=\left[1+\left(R_{100}-R\right)\left(R-R_{0}\right)^{-1}\left(P_{100} / P_{0}\right)\right]^{-1}$, where $P_{100}$ and $P_{0}$ are the counts in the peak corresponding to $R_{100}$ and $R_{0}$. In Fig. $4, F_{0}$, the Ps fraction 


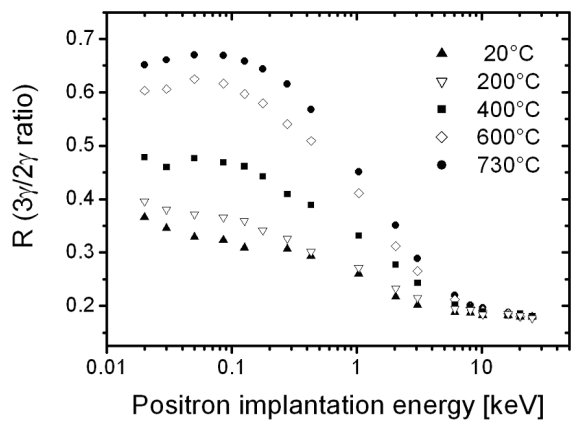

Fig. 3. $3 \gamma / 2 \gamma$ annihilation ratio ( $R$ parameter) as a function of positron implantation energy in a Ge crystal at different temperatures $\left(200-730^{\circ} \mathrm{C}\right)$.

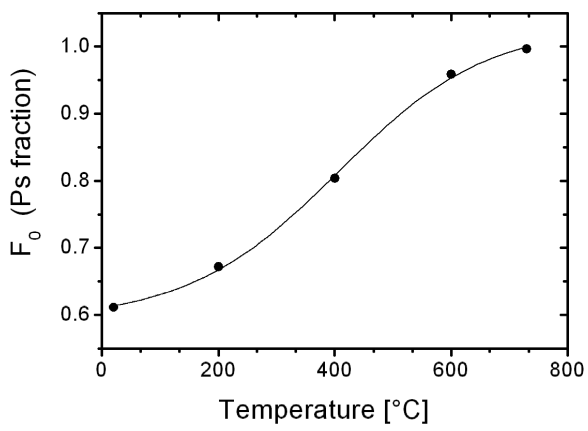

Fig. 4. Positronium fraction $\left(F_{0}\right)$ extrapolated at zero positron implantation energy, as a function of the temperature of the Ge crystal.

at zero energy, is reported as a function of the Ge sample temperature. The value of $F_{0}$ increases with $T$ and saturates for $T>700^{\circ} \mathrm{C}$.

The $R_{n}$ parameter obtained by calibration is related to the positronium fraction $F$ formed in the medium, $\lambda_{3 \gamma}$ the $o$-Ps annihilation rate and the pick-off rate $\lambda_{\text {p.o. }}[19,32]$ :

$$
R_{n}=F\left[\lambda_{3 \gamma} /\left(\lambda_{3 \gamma}+\lambda_{\text {p.o. }}\right)\right] .
$$

If $F$ is known, $\lambda_{\text {p.o. }}$ can be calculated and by the Eldrup-Tao model $[33,34,11]$ an estimation of pore sizes can be obtained. For the as-deposited sample of Fig. 1, supposing that all positrons in the film are trapped in pores and form positronium, a diameter $d \geq 1.2 \mathrm{~nm}$ was found.

Analysis of the $3 \gamma$-PAS curves allows establishing the pore interconnectivity threshold by extracting the Ps escape length from the data [6]. Such analysis is based on a model first proposed to study Ps formation and out-diffusion in ice [35]. As a case study we present in Fig. $5 \mathrm{a}$ and b the $S$ and the $R_{n}$ curves for a-C:N:H samples produced by PECVD at different bias voltage. The sample produced with the lowest bias voltage is less dense, as expected, has a very high $S$ value and gives 


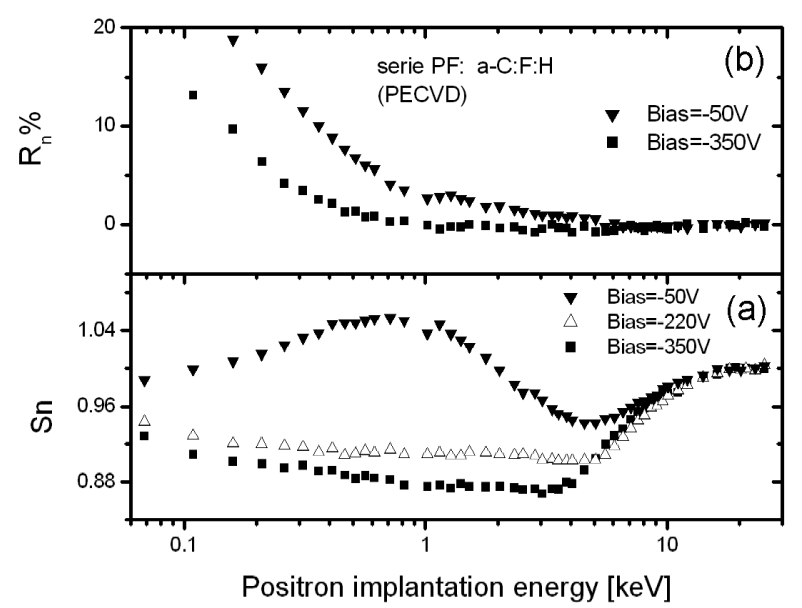

Fig. 5. (a) $S_{n}$ parameter and (b) $R_{n}$ parameter as a function of depth for a-C:N:H samples at three different biases.

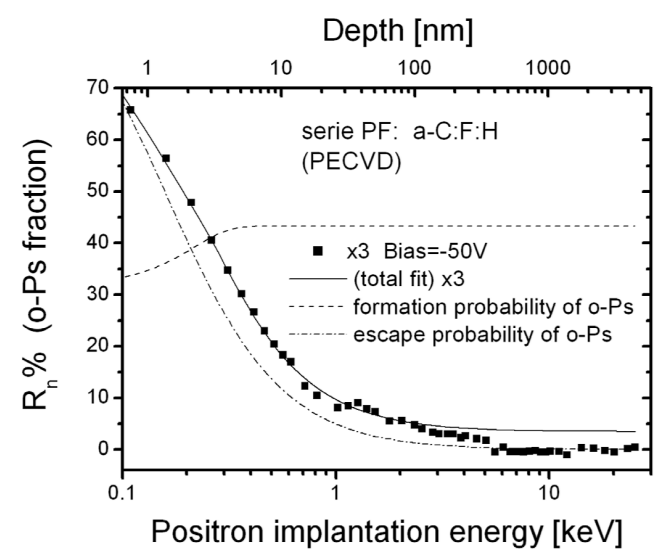

Fig. 6. $R_{n}$ parameter as a function of positron implantation energy (lower scale) and mean positron implantation depth (upper scale) for the a-C:N:H sample at $-50 \mathrm{~V}$ bias. The lines are the fit to the data.

a small signal of $3 \gamma$ annihilation. In Fig. 6 the $3 \gamma$ signal is reported with the fit obtained by the model. On the figure the two lines indicating $o$-Ps formation and $o$-Ps escaping are also shown. The $o$-Ps diffunsion length $L_{o-P s}$, in the first approximation [6], can be related to the positron diffusion length $L_{+}$and the $o$-Ps escaping length $L_{\mathrm{esc}}$ : $L_{o-\mathrm{Ps}}^{2}=L_{\mathrm{esc}}^{2}+L_{+}^{2}$. In this case the found Ps escaping length, due to the similar $L_{+}$and $L_{o-\mathrm{Ps}}$, is less than $1 \mathrm{~nm}$, and points out that there are not connected pores toward the surface. From $F=0.4$ (see Fig. 6) and Eq. (3), a diameter $d \geq 1 \mathrm{~nm}$ of the pores is estimated. The amorphous carbon film is rich of small pores and open volume defects. 


\section{Conclusions}

DP-PAS, with its different measurement techniques, is demonstrated to be a powerful tool for porosity characterization of low- $\kappa$ materials. DBS and $3 \gamma$-PAS can give information on distribution of pores, closed and open porosity, percolation threshold and chemical environment around the pores. From DBS and $3 \gamma$-PAS, only an estimation of the size of the pores can be gained. On the contrary PALS can give size distribution of the pores. DP-PAS, due to the need of complex apparatuses and the lack of facilities and laboratory with slow positron beams, cannot be seen at present as a standard technique for microelectronic industry. On the contrary, it is a very important analytical technique for advanced research in developing new materials with very low $\kappa$. DP-PAS is particular suited for studying the size distribution of pores and probing the presence of smallest pores.

\section{Acknowledgments}

We thank E. Carollo and M. Bacchetta (ST Microelectronics-Agrate-Milano-Italy) for $\mathrm{SiOCH}$ samples and F.L. Freire Jr. for a-C:N:H samples.

\section{References}

[1] International Technology Roadmap for Semiconductors (2003), http://public.itrs.net/Files/2003ITRS/Home2003.htm.

[2] B. Li, T.D. Sullivan, T.C. Lee, D. Badami, Microelectron. Reliab. 44, 365 (2004).

[3] K. Maex, M.R. Baklanov, D. Shamiryan, F. Iacopi, S.H. Brongersma, Z.S. Yanovitskaya, Appl. Phys. 93, 8793 (2003).

[4] D.W. Gidley, K.G. Lynn, M.P. Petkov, M.H. Weber, J.N. Sun, A.F. Yee, in: New Directions in Antimatter Chemestry and Physics, Eds. C.M. Surko, F.A. Gianturco, Kluwer Academic Publishers, Dordrecht (The Netherlands) 2001, p. 151.

[5] T.L. Dull, W.E. Frieze, D.W. Gidley, J.N. Sun, A.F. Yee, J. Phys. Chem. B 105, 4657 (2001).

[6] M.P. Petkov, C.L. Wang, M.H. Weber, K.G. Lynn, K.P. Rodbell, J. Phys. Chem. B 107, 2725 (2003).

[7] K.P. Mogilnikov, M.R. Baklanov, D. Shamiryan, M.P. Petkov, Jpn. J. Appl. Phys. 43, 247 (2004).

[8] M.P. Petkov, M.H. Weber, K.G. Lynn, K.P. Rodbell, S.A. Cohen, Appl. Phys. Lett. 74, 2146 (1999).

[9] M.P. Petkov, M.H. Weber, K.G. Lynn, K.P. Rodbell, Appl. Phys. Lett. 79, 3884 (2001).

[10] M.P. Petkov, M.H. Weber, K.G. Lynn, K.P. Rodbell, Appl. Phys.Lett. 77, 2470 (2000).

[11] D.W. Gidley, W.E. Frieze, T.L. Dull, J. Sun, A.F. Yee, C.V. Nguyen, D.Y. Yoon, Appl. Phys. Lett. 76, 1282 (2000).

[12] A. Uedono, Z. Quan Chen, R. Suzuki, T. Ohdaira, T. Mikado, S. Fukui, A. Shiota, S. Kimura, J. Appl. Phys. 90, 2498 (2001). 
[13] M.P. Petkov, M.H. Weber, K.G. Lynn, K.P. Rodbell, S.A. Cohen, J. Appl. Phys. 86, 3104 (1999).

[14] K. Ito, Y. Kobayashi, K. Hirata, H. Togashi, R. Suzuki, T. Ohdaira, Radiat. Phys. Chem. 68, 435 (2003).

[15] Y. Kobayashi, W. Zeng, T.B. Chang, K. Hirata, R. Suzuki, T. Ohdaira, K. Ito, J. Appl. Phys. 91, 1704 (2002).

[16] R.S. Yu, K. Ito, K. Hirata, W. Zheng, Y. Kobayashi, J. Appl. Phys. 93, 3340 (2003).

[17] R.S. Yu, K. Ito, K. Hirata, K. Sato, W. Zheng, Y. Kobayashi, Chem. Phys. Lett. 379, 359 (2003).

[18] R. Suzuki, T. Ohdaira, Y. Kobayashi, K. Ito, Y. Shioya, T. Ishimura, Radiat. Phys. Chem. 68, 339 (2003).

[19] R.S. Brusa, M. Spagolla, G.P. Karwasz, A. Zecca, G. Ottaviani, F. Corni, M. Bacchetta, E. Carollo, J. Appl. Phys. 95, 2348 (2004).

[20] R.S. Brusa, C. Macchi, S. Mariazzi, M. Spagolla, G.P. Karwasz, A. Zecca, Mater. Sci. Forum 445-446, 268 (2004).

[21] C. Macchi, G. Mariotto, A. Zecca, M. Bettonte, R.S. Brusa, Material. Science and Semiconductor Processing 4-6, 289 (2004).

[22] Y. Hayashi, J. Kawahara, K. Shiba, M. Tagamy, S. Saito, T. Onodera, K. Kinoshita, M. Hiroi, NEC. Res. Dev. 42, 51 (2001).

[23] H.-J. Lee, C.L. Soles, D. Liu, B.J. Bauer, E.K. Lin, W. Wu, A. Grill, J. Appl. Phys. 95, 2355 (2004).

[24] R.S. Brusa, G.P. Karwasz, M. Bettonte, A. Zecca, Appl. Surf. Sci. 116, 59 (1997).

[25] A. Zecca, M. Bettonte, J. Paridaens, G.P. Karwasz, R.S. Brusa, Meas. Sci. Technol. 9, 1 (1998).

[26] R.S. Brusa, G.P. Karwasz, N. Tiengo, A. Zecca, F. Corni, R. Tonini, G. Ottaviani, Phys. Rev. B 61, 10154 (2000).

[27] M. Clement, J.M.M. de Nijs, P. Balk, H. Shut, A. van Veen, J. Appl. Phys. 79, 9029 (1996).

[28] Positron and Positronium Chemistry, Eds. D.M. Schrader, Y.C. Jean, Elsevier Sci. Publ., Amsterdam 1988.

[29] A.P. Mills Jr., Phys. Rev. Lett. 41, 1828 (1978).

[30] A.P. Mills Jr., p. 432 and G.K. Lynn p. 609 in: Positron Solid State Physics, Eds. W. Brandt, A. Dupasquier, North Holland Publ., Amsterdam 1983.

[31] E. Soininen, A. Schwab, K.G. Lynn, Phys. Rev. B 43, 10051 (1991).

[32] R.S. Brusa, G.P. Karwasz, G. Mariotto, A. Zecca, R. Ferragut, P. Folegati, A. Dupasquier, G. Ottaviani, R. Tonini, J. Appl. Phys. 94, 7483 (2003).

[33] M. Eldrup, in: Positron Annihilation, Eds. P.G. Coleman, S.C. Sharma, L.M. Diana, North Holland Publ., Amsterdam 1982, p. 753.

[34] H. Nakanishi, Y.C. Jean, in: Positron and Positronium Chemistry, Eds. D.M. Schrader, Y.C. Jean, Elsevier Sci. Publ., Amsterdam 1988, p. 159.

[35] M. Eldrup, A. Vehanen, P.J. Shultz, K.G. Lynn, Phys. Rev. B 32, 7048 (1985). 